Ann. Génét. Sél. anim., I977, 9 (I), 63-72.

\title{
Choix et pondération économique des caractères en sélection animale ${ }^{(1)}$
}

\author{
W. SCHLOTE \\ Institut für Tierhaltung und Tierzüchtung, Universität Hohenheim \\ Postfach Io6 (06 300), D-7000 Stuttgart 70
}

\begin{abstract}
Résumé
Deux problèmes liés à la sélection sur un indice de plusieurs caractères sont discutés, le choix des caractères à inclure dans la valeur génétique globale et les coefficients de pondération économique de ces traits. Les conditions nécessaires à la prise en compte d'un caractère dans l'objectif de sélection ainsi que différentes méthodes de calcul des poids économiques sont données. Le critère de comparaison des caractères est leur importance génético-économique. Cette valeur est calculée comme le produit du poids économique et de l'écart type génétique du caractère, compte tenu des réponses indirectes sur les autres traits dues à la sélection sur ce caractère. Des exemples chez le porc et les bovins sont présentés et le choix d'une combinaison de plusieurs caractères pour la valeur génétique globale est montrée. La répartition du profit dû à la sélection entre les groupes intéressés par celle-ci ainsi que la définition de l'objectif de la sélection font l'objet principal de la discussion.
\end{abstract}

\section{I. - Introduction}

L'amélioration génétique des animaux domestiques n'est pas une simple étude académique. Les agriculteurs sont condamnés à survivre dans un milieu de concurrence économique et on a cessé de surestimer le standard des races pour insister sur les caractères d'importance économique : l'objectif de la sélection est de fournir aux éleveurs des animaux dont ils peuvent retirer le maximum de profit.

La valeur économique des animaux dépend en général de plusieurs caractères qu'il faudrait tous améliorer pour atteindre le profit maximum ou les coûts minimum. Pour sélectionner sur plusieurs caractères, on utilise classiquement un indice linéaire (SMITH, I936; HAZEL, I943). Cependant le progrès génétique attendu pour un caractère particulier quand on utilise un indice de $n$ traits n'est qu'une

(1) Cet article a fait l'objet d'une communication lors du séminaire sur " L'estimation de la valeur génétique des reproducteurs " organisé à Toulouse les 6 et 7 octobre 1976 par le Département de Génétique Animale de l'I.N.R.A. (France). 
fraction du progrès qui est obtenu quand seul ce caractère est sélectionné, cette fraction égalant $\mathrm{I} / \sqrt{n}$ si les traits sont indépendants et si le produit de leur poids économique, leur héritabilité et leur écart-type est égal (HAZEL et LusH, I942). Il est donc nécessaire de limiter le nombre de caractères dans l'indice de sélection, c'est pourquoi on ne choisira que ceux qui sont les plus importants.

Notre objet est de discuter ce choix ainsi que la pondération économique des variables retenues dans l'indice.

\section{II. - Les critères pour le choix des' caractères}

Il est évident que pour la sélection des bovins laitiers la production de lait est un caractère très important, alors qu'il est inutile d'inclure la forme des oreilles des porcs dans un indice. Pour beaucoup de caractères la décision est rarement aussi facile et on a besoin de critères précis. Par ailleurs, au-delà de l'importance économique de la variable, il faudra tenir compte des possibilités de progrès génétique sur celle-ci. Dès lors, un caractère doit remplir deux conditions pour être pris en considération dans un indice (Heidhues, I962) : il faut que ce caractère :

I) contribue substantiellement au rendement économique (coefficient économique);

2) soit améliorable génétiquement (héritabilité, variabilité génétique).

Le second point étant largement discuté dans la littérature, nous insisterons sur la valeur économique des caractères.

\section{III. - Méthodes de calcul des poids économiques}

L'utilisation de pondérations économiques a été proposée par Smith dès I936. Plusieurs méthodes sont envisageables pour les estimer.

\section{I. - Estimation subjective}

Achilles (ig68) ainsi que Breloh et Schmitten (I970) pour la qualité de la viande chez le porc et FEwson (I976) pour la forme de la mamelle chez les bovins ont donné des exemples d'une telle estimation. Il est évident qu'un calcul économique exact serait préférable. Mais dans certains cas - tels que ceux abordés dans les travaux cités - un tel calcul est à peine possible. Le poids qu'on veut attribuer à un caractère est alors estimé relativement à celui d'une autre caractère important. C'est d'ailleurs la méthode utilisée initialement par Smith (I936).

\section{2. - Calcul du profit marginal}

L'importance économique d'un caractère pour la sélection peut être exprimé par le profit marginal qui résulte de l'augmentation d'une unité de ce caractère au-dessus de la moyenne de la population (HAZEL, I943). Le profit marginal est calculé par la différence entre recettes marginales et frais marginaux. On peut distinguer plusieurs situations différentes. 
Pour certains caractères l'amélioration consiste en une augmentation marginale des recettes sans modification des coûts de production. Cette situation est en particulier celle des caractères de qualité quand le prix des produits sur le marchó varie avec le niveau de ces caractères. Dans ce cas le poids économique sera la variation de prix par unité d'écart-type du caractère. Il en est ainsi de la proportion de morceaux nobles dans la carcasse chez le porc (BöckenHoff et al., I967) et chez les bovins (ADELhelm et al., I972) ainsi que du rendement commercial chez les bovins (SCHLOTE et Fewson, I972; Schlote, I974.).

La deuxième situation, similaire à la première, est celle où la sélection a pour effet de réduire les coûts sans modifier les recettes. C'est en particulier le cas de la consommation d'aliment chez le porc (Böc KenHoff et al., I967; Ollivier, I97I; MaRguARDT, 1974) et chez les bovins (ZEDDIES, I973). Le profit marginal est donc ici le prix de la quantité d'aliment économisée par rapport à la moyenne de la population.

Dans la situation classique envisagée par HAZEL (I943), les prix des facteurs produits et ceux des facteurs consommés sont connus (prix du marché). Le profit marginal ou le poids économique pour un caractère peut alors être calculé par la formule (ADELHELm et al., I972) :

$$
a=\sum_{i=1}^{k} q_{i} p_{i}-\sum_{j=\mathbf{I}}^{m} q_{j} p_{j}
$$

où

a est le coefficient de pondération économique

$q_{i, j} \quad$ la quantité supplémentaire de facteur $i$ produit ou $j$ consommé, respectivement, du fait d'accroissement d'une unité du caractère sélectionné,

$p_{i, j} \quad$ et les prix commerciaux unitaires des facteurs $i$ et $j$.

Par exemple, l'accroissement du nombre de porcelets de neuf à dix par portée augmente la valeur de celle-ci de 53,60 DM (valeur du porcelet supplémentaire). Par contre la truie et le dixième porcelet consomment en plus $30,2 \mathrm{~kg}$ d'orge (à $0,436 \mathrm{DM} / \mathrm{kg}$ ) et $7,5 \mathrm{~kg}$ de farine de poisson (à $0,75 \mathrm{DM} / \mathrm{kg}$ ), le mélange de ces $37,7 \mathrm{~kg}$ de farine coûtant $0,035 \mathrm{DM} / \mathrm{kg}$, et les frais vétérinaires s'accroissent de 25 DM (BöcKenhoff et al., I967).

Le poids économique du nombre de porcelets s'obtient alors en divisant ces coefficients par la taille initiale de la portée (9) :

$$
\begin{aligned}
a= & 0, \mathrm{II} \times \mathrm{I} \times 53,60 \mathrm{DM}-(0, \mathrm{II} \times 30,2 \mathrm{~kg} \times 0,436 \mathrm{DM}+0, \mathrm{II} \times 7,5 \mathrm{~kg} \times 0,750 \\
& \mathrm{DM}+0, \mathrm{II} \times 37,7 \mathrm{~kg} \times 0,035 \mathrm{DM}+0, \mathrm{II} \times 2,50 \mathrm{DM}) \\
& =3,74 \mathrm{DM} .
\end{aligned}
$$

Cette méthode de calcul a été utilisée par beaucoup d'auteurs four des caractères différents (HAzel, I943; SOLler et al., I966; BöCKENHOFF et al., r967; Adelhelm et al., i972; Haring, i972; Zeddies, I973; McClintock et CunninGHAM, I974).

Il n'est souvent pas possible d'obtenir directement certains prix ou coûts unitaires. Dans ce cas les économistes utilisent des valeurs indirectes telles que les valeurs de substitution ou les coûts d'opportunité.

Dans chaque entreprise, que ce soit une exploitation agricole ou une économie nationale, certains facteurs de production sont fixés, telles que les surfaces utilisables. L'amélioration d'un caractère a des effets sur la valorisation du facteur et 
peut changer l'organisation optimale de l'entreprise. Par programmation linéaire, on peut trouver la meilleure utilisation des facteurs de production fixés et en déduire la contribution optimale de la spéculation au profit de l'entreprise.

Par transformation de l'accroissement du profit de l'entreprise dû à l'amélioration d'un caractère, on obtient le poids économique du caractère en question. Bien qu'elle soit longue, cette méthode est la plus rigoureuse. Adelhelm et al. (I972) ainsi que HARING (I972) ont utilisé la programmation linéaire pour calculer des coefficients économiques.

\section{3. - Régression sur les recettes}

Plusieurs auteurs ont calculé les poids économiques par régression partielle du caractère sur les recettes, les coûts ou la différence des deux (GRAVERT et Rosenhahn, I965; Rittler et al., ig66; Spahr, i967; Pearson et al., i97o; Ollivier, I97 I; MarquardT, I974). Pour appliquer cette méthode il faut connaître la valeur économique d'un grand nombre d'animaux. Par ailleurs les pondérations sont estimées d'après la situation économique actuelle et cette méthode a par conséquent le désavantage de ne pas considérer l'avenir qui est pourtant fondamental du point de vue de la génétique et de la sélection. En outre la valeur économique d'un caractère calculée par régression dépend des autres variables considérées. La contribution d'un caractère au profit n'est plus basée sur des relations causales ce qui limite la valeur de cette méthode.

\section{4. - Choix des poids économiques selon le progrès atteint}

Dans certaines situations où le calcul direct du profit marginal lié à l'amélioration d'un caractère particulier n'est pas possible, on peut être conduit à l'application de cette méthode d'approximations successives. On calcule le progrès génétique pour chacun des caractères introduit dans l'indice en donnant un poids initial au caractère concerné. Puis on fait varier le poids de ce caractère jusqu'à ce que les progrès génétiques calculés pour tous les caractères se trouvent dans un optimum subjectif. Cette méthode a été appliquée notamment pour la pondération de la qualité de la viande chez le porc (FlOCK, I968; MARquardT, I974; Fender et al., I976). Les indices de sélection avec restrictions sont d'ailleurs seulement des variantes de la méthode avec un objectif défini plus rigoureusement.

Proche de l'estimation subjective des poids économiques, cette méthode a l'avantage d'être basée sur un critère quantitatif, le progrès génétique.

\section{IV. - Choix des caractères à sélectionner}

\section{I. - Évaluation d'un seul caractère}

Les poids économiques étant calculés, il faut donc décider quels sont les caractères qu'on considère dans la valeur génétique globale. La variabilité des caractères différant beaucoup de l'un à l'autre, on ne peut pas comparer les poids économiques des caractères directement. Il faut les rapporter à leur écart-type. Le généticien s'intéresse plutôt à l'exploitation de la variation génétique additive. 
Le critère de mesure de l'intérêt de la sélection d'un caractère particulier sera par conséquent le produit du poids économique et de l'écart type génétique additif de ce caractère (FEWSON et al., I967; NiEBEL et al., I972; BöcKenHofF et al., I976). Cette valeur exprime le profit marginal lié à un progrès génétique direct d'un écart-type de ce caractère. Mais la sélection sur ce caractère engendre un progrès génétique indirect sur les autres caractères, donc un profit ou une perte supplémentaire. On peut calculer la valeur de ce progrès indirect par la formule (FEWSON et al., I967):

où

$$
c_{\mathrm{A}_{i}}=\sum a_{j} \sigma_{\mathrm{A}_{j}} \gamma_{\mathrm{P}_{i} \mathrm{~A}_{j}}
$$

$c_{\mathrm{A}_{i}} \quad$ le profit supplémentaire indirect pour le caractère $i$,

$a_{j} \quad$ le poids économique pour le caractère $j$ lié au caractère $i, j=\mathrm{I}, \ldots, k$,

$\sigma_{\hat{A}_{j}} \quad$ l'écart type génétique additif du caractère $j$,

$r_{A_{i} A_{j}} \quad$ la corrélation génétique additive entre les caractères $i$ et $j$.

Le critère pour juger l'importance génético-économique des caractères est alors la somme des plus-values génético-économiques directes et indirectes $\left(a_{i} \sigma_{\mathrm{A}_{i}}+c_{\mathrm{A}_{i}}\right)$. A titre d'exemple des valeurs de ces critères sont données pour le porc (FENDER et al., I976) et pour les bovins de race mixte (NIEBEL et al., I972).

\section{TABLEAU I}

L'importance économique de différents caractèves chez le porc en DM par animal

(Fender et al., I976, Résultats préliminaires)

Economic importance of different characters in pig, in DM per heal

(FENDER et al., 1976, preliminary results)

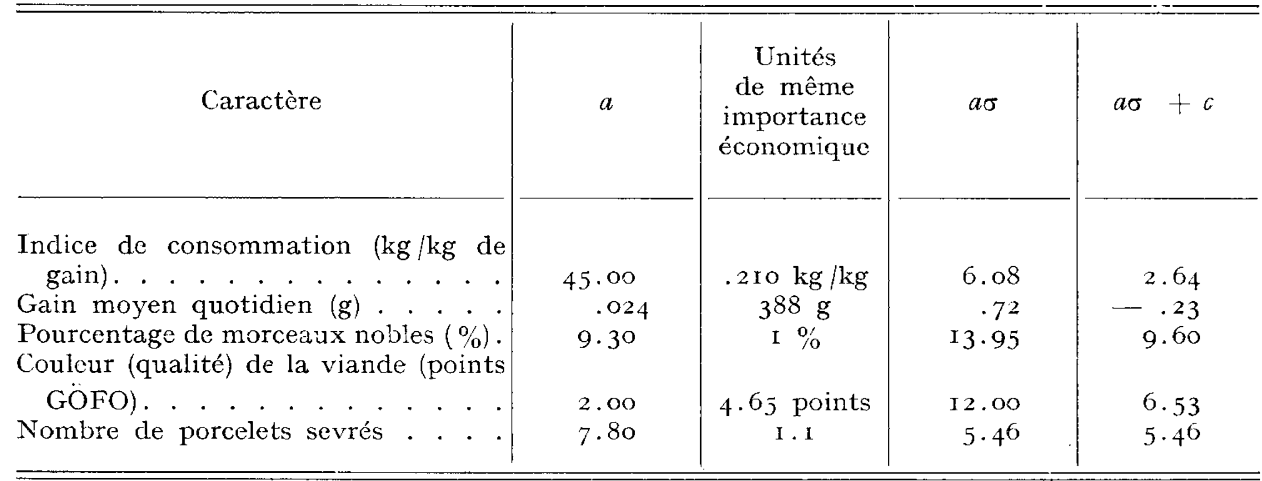

Chez le porc (tableau I), l'indice de consommation a un poids beaucoup plus élevé que le gain moyen quotidien. Les coefficients du pourcentage de morceaux nobles et d’ nombre de porcelets sevrés sont à un niveau moyen tandis que les poids économiques du gain moyen quotidien et de la couleur de la viande sont assez bas. Dans la deuxième colonne, on montre les progrès génétiques en chacun des caractères qui apportent la même augmentation de profit qu'une plus value de I p. Ioo en pourcentage de morceaux nobles. L'importance génético-économique ( $3^{\mathrm{e}}$ colonne) est assez différente du poids économique. Selon ca critère, le pourcen- 
tage de morceaux nobles et la couleur de la viande sont les caractères les plus importants pour la sélection. Les espérances des gains économiques pour l'indice de consommation et le nombre de porcelets sont moins bonnes et l'amélioration du gain moyen quotidien est encore inférieur. Les progrès génétiques indirects entraînent presque toujours une réduction du profit $\left(4^{\mathrm{e}}\right.$ colonne) car les réponses corrélatives sont négatives. Ccci vient de ce que, dans le cas d'une alimentation ad libitum, les corrélations entre caractères sont trouvées pour la plupart négatives. C'est ce profit total qui est le meilleur critère de choix des caractères à prendres en considération dans la valeur génétique globale.

TABLEAU 2

L'importance économique de différents caractères chez les bovins de race mixte en DM par unité standard (d'après NiEBEL et al., I972)

Economic importance of aifferent characters in dual purpose cattle in D.I per standard unit (from NIEBEL et al., I972)

\begin{tabular}{|c|c|c|c|c|c|}
\hline Caractère & Unité & $a$ & $\begin{array}{l}\text { Unités } \\
\text { de même } \\
\text { importance } \\
\text { économique }\end{array}$ & $a \sigma_{\mathrm{A}}$ & $a \sigma_{\mathrm{A}}+c_{\mathbf{A}}$ \\
\hline Quantité de lait sans matière & & & & & \\
\hline grasse. . . . . . . . . . . . & I OO $\mathrm{kg}$ & $2 \cdot 3.5$ & I $166 \mathrm{~kg}$ & 7 & 295 \\
\hline Quantité de matière grasse. & I kg & $27 \cdot 4 \mathrm{r}$ & I $\mathrm{kg}$ & $37^{\circ}$ & 368 \\
\hline Consommation d'aliment . . & $\begin{array}{l}\text { Ioo valeurs } \\
\text { amidon }\end{array}$ & 59.00 & $\begin{array}{l}46 \text { valeurs } \\
\text { amidon }\end{array}$ & 93 & 208 \\
\hline $\begin{array}{l}\text { Gain moyen quotidien . . . } \\
\text { Capacité de croissance - poids }\end{array}$ & I OO $\mathrm{g}$ & 130.00 & $2 \mathrm{Ig}$ & $6 \tilde{j}$ & 208 \\
\hline $\begin{array}{l}\text { final possible. } \cdot \cdot \cdot \cdot \cdot \cdot \cdot \cdot \cdot \\
\text { Pourcentage de morceaux no- }\end{array}$ & IOO kg & I 54.00 & I $8 \mathrm{~kg}$ & 54 & 225 \\
\hline bles. . . . . . . . . . & I $\%$ & $24 \cdot 40$ & I. I \% & I 5 & I5 \\
\hline Intervalle entre vêlages. . . & Io jours & I 47.00 & I. 9 jours & I 32 & I9 \\
\hline Longévité . . . . . . . . . . & I lact. & 202.00 & I 4 lact. & 127 & I 27 \\
\hline
\end{tabular}

En ce qui concerne les bovins (tableau 2) les pondérations ont été calculées à partir du profit retiré par l'éleveur de l'exploitation d'une vache faisant 5 lactations ainsi que de toute sa descendance. Les hypothèses sousjacentes à l'estimation de ces valeurs sont une étable de 40 vaches, un haut niveau de production, un prix de la viande élève, des prix du lait et de la farine moyens. Le caractère qui a le poids économique le plus élevé (colonne 2) est la longévité tandis que le poids de la quantité de matière grasse est très faible. Dans la troisième colonne figurent les équivalences d'un point de vise économique avec I $\mathrm{kg}$ de matière grasse. Les intérêts de la quantité de matière grasse et de la longévité sont inversés quand on se place du point de vue génético-économique (4 ${ }^{\mathrm{e}}$ colonne). La quantité de matière grasse cst alors le caractère le plus important. Ceci reste vrai quand on raisonne sur le profit total (dernière colonne), qui tient compte des effets indirects de la sélection. 


\section{2. - Évaluation d'une combinaison de plusieurs caractères}

La meilleure valeur génétique globale est évidemment celui qui tient compte de tous les caractères. Mais nous avons dit plus haut que dans le cas d'une sélection sur un indice, le progrès sur un caractère dépend du nombre de caractères considérés. Alors on peut risquer un progrès génétique dans des caractères majeurs par l'inclusion de beaucoup de caractères mineurs. D'un point de vue pratique il est en outre plus facile d'avoir peu de caractères dans un indice. On est alors tenté de se limiter aux caractères les plus importants. Il faut cependant recalculer les poids économiques à appliquer dans la valeur génétique réduite, en tenant compte de ?'effet de la suppression des caractères, qui étaient pris en compte dans la valeur génétique globale sur les pondérations des variables retenues.

Les nouvelles pondérations peuvent être estimées à partir des anciennes grâce à la relation suivante (modifiée d'après Niebel et al., 1972) :

$$
\mathrm{G}_{\mathrm{K}} a_{\mathrm{R}}=\mathrm{G}_{\mathrm{RT}} a_{\mathrm{T}}
$$

où $a_{\mathrm{R}} \quad$ est le vecteur des poids économiques dans la valeur génétique réduite, $a_{\mathrm{T}} \quad$ le vecteur des poids économiques dans la valeur génétique globale,

$G_{R} \quad$ la matrice des variances et covariances génétiques pour les caractères retenus et

$\mathrm{G}_{\mathrm{RT}} \quad$ la matrice des variances et covariances génétiques pour les caractères initiaux (colonnes) et retenus (lignes).

TABLEAL 3

Valeurs de $\sigma_{\mathrm{A}_{\mathrm{T}}{ }^{\prime}}$ et $\mathrm{r}_{\mathrm{ATA}_{\mathrm{T}} \mathrm{T}^{\prime}}$ pour les combinaisons successives optimales des caractères chez les bovins (NiEBel et al., 1972)

Values of $\sigma_{\mathrm{A}_{\mathrm{T}}}$ and $\mathrm{r}_{\mathrm{A}_{\mathrm{T}} \mathrm{A}_{\mathrm{T}}}$ for successive optimal combinations of characters in cattle (Niebel et al., 1972)

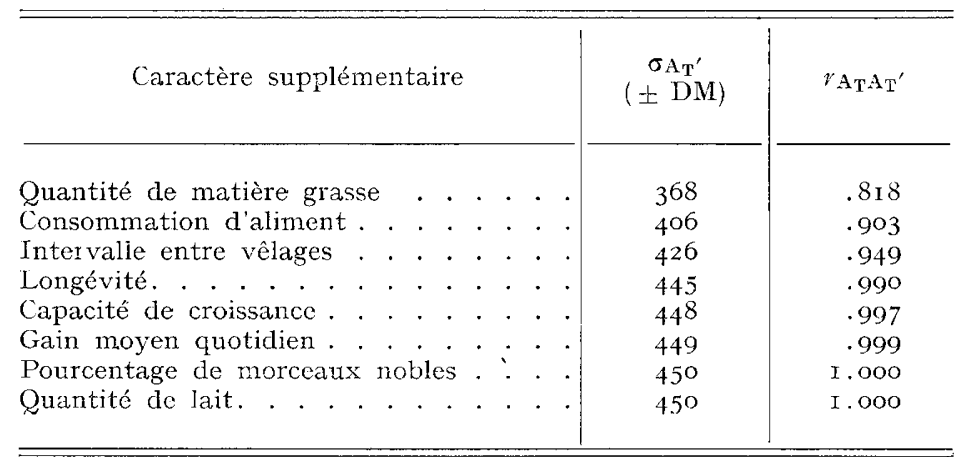

Les critères de choix des caractères à retenir dans l'index réduit sont évidemment l'écart-type de la valeur génétique réduite $\left(\sigma_{\mathbf{A r}^{\prime}}\right)$ et la corrélation entre la valeur génétique réduite et la valeur génétique globale $\left(r_{\mathrm{ATAT}}\right)$. Alors on retient ces caractères dans la valeur génétique globale dont la combinaison donne les valeurs maximum pour l'écart-type $\sigma_{\mathrm{AT}}$ et la corrélation $v_{\mathrm{ATAT}}$. Par un procédé successif on commence avec le caractère qui a la plus grande importance génético-écono- 
mique $\left(a \sigma_{\mathrm{A}}+c_{\mathrm{A}}\right)$, valeur qui est égale à $\sigma_{\mathrm{Ar}^{\prime}}$ pour un seul caractère. Puis on ajoute ce caractère qui augmente l'écart-type génético-économique le plus parmi tous les combinaisons à deux avec le premier caractère.

Niebel et al. (I972) ont déterminé, dans le cas des bovins, les valeurs génétiques réduites définies successivement pour un nombre croissant de caractères et qui maximisent les critères de choix. La précision est très bonne dès qu'on introduit deux caractères. On pourrait alors sélectionner les bovins de race mixte une valeur génétique globale ne comprenant que les deux caractères les plus importants : quantité de matière grasse et consommation d'aliment, le gain moyen quotidien pouvant d'ailleurs se substituer à ce dernier trait sans perte de précision.

\section{V. - Discussion et Conclusion}

La détermination des valeurs exactes des coefficients de pondération des caractères dans la valeur génétique globale est un problème toujours difficile et les auteurs ont souvent utilisées des méthodes sous optimales pour y parvenir. Par ailleurs les valeurs qu'ils obtiennent sont évidemment dépendantes des conditions économiques propres à leur pays, en particulier pour les niveaux de prix et de coûts. En outre la définition de ces coûts varie avec le type de production. Il n'est donc possible ni de comparer les résultats obtenus par chacun d'entre eux, ni de donner des recettes générales.

D'autre part, l'unité de production à partir de laquelle est définie la valeur génétique globale varie avec les auteurs. Si le problème ne se pose pas chez le porc pour lequel le produit final est unique, il existe pour les bovins ou la valeur génétique globale a été défini comme celle du descendant moyen (SOLLER et al., I966), de la vache faisant 5 lactations ainsi que toute sa descendance (ADELHELm et al., I972; ZEDDIES, I973), de la vache par an (HARING, I972), de l'insémination moyenne (MCClintock et Cunningham, I974). Si, dans une étude particulière, l'unité de référence reste la même, son choix est de peu d'importance à partir du moment ou les profits marginaux dus à l'amélioration des différents caractères sont correctement actualisés. Cette actualisation est en particulier nécessaire quand ces profits marginaux ne s'expriment pas aux mêmes moments (Soller et al., ig66; McClintock et Cunningham, I974; Niebel, I974). En définitive il est encourageant de constater que les pondérations économiques s'accordent assez bien malgré des situations économiques différentes, des méthodes de calculs différentes.

D'autres problèmes sont liés à l'objectif de la sélection dont nous avons dit qu'il était d'accroître le profit de l'éleveur. En premier lieu, les effets de la sélection ne sont sensibles qu'à long terme puisqu'il faut une longue période (5 à I5 ans) avant d'observer un progrès génétique en production animale. Il faudrait alors, dans le calcul des pondérations économiques, tenir compte des rapports de prix futurs, ce qui est généralement très difficile. Dans certains cas cependant l'évolution des prix reste stable sur de longues périodes. Quoiqu'il en soit, il est indispensable de prondre en considération cet aspect prospectif quand on raisonne sur la sélection.

Un second problème fondamental est celui de la répartition du profit dû au progrès génétique. Trois groupes sont intéressés par ce profit (HARRIS, I9jo; ZEDdies et WENIGER, I970; ZEDDIES, I973) :

- les agriculteurs, 
- les entreprises de sélection (associations herd-book, entreprises commerciales, sélectionneurs),

- l'économie nationale ou supra-nationale (les consommateurs).

S'il est décevant de constater que les agriculteurs ne perçoivent généralement qu'une faible part du profit dû à la sélection, on constatera que le progrès génétique peut les aider à maintenir leur niveau de production. Du fait de la concurrence les prix des produits n'augmentent pas mais les coûts diminuent et la qualité s'améliore. L'économie nationale et les consommateurs profitent alors automatiquement du progrès génétique. De plus on comprend bien que, dans la mesure où il investit dans la sélection, l'État désire en définir l'objectif au profit du consommateur. Les sélectionneurs et leurs associations l'ont d'ailleurs toujours fait selon leur intérêt. Dans une situation de concurrence à tous les niveaux de production, ces oppositions sur l'objectif de la sélection disparaîtraient. Cependant cet état de concurrence n'existe probablement pas et il faut toujours tenir compte de cette divergence d'intérêt. Les poids économiques montrés dans les tableaux I (FENDER et al., I976) et 2 (NIEBEL et al., I972) sont d'ailleurs calculés du point de vue des agriculteurs, mais sous l'hypothèse de concurrence. Il faut aussi noter que celui qui investit dans la sélection animale et qui prend les décisions pour la sélection, doit définir son propre objectif, donc la valeur génétique globale et les poids économiques. Le rôle de la recherche scientifique est alors seulement de développer les méthodes de calcul des poids économiques.

Enfin, on pourrait tout aussi bien étendre cet objectif du niveau économique au niveau socio-économique (préservation des structures sociales, exploitation des surfaces marginales, etc...) ou du long terme au très long terme (préservation des gènes, etc...).

Toutefois les méthodes mathématiques nécessaires à ces extensions ne sont pas encore au point. D'autre part, il ne faut pas oublier que toute extension de l'objectif peut réduire le progrès génétique et le gain économique possible. Enfin, il y a maintenant des méthodes pour définir l'objectif de sélection et les poids économiques, les entreprises de sélection ne devraient pas hésiter à les appliquer dans leur travail.

Rę̧ pour putlication en férnier 1977.

\section{Remerciements} manuscrit.

Je remercie J. M. Elsen et J. L. Foulley pour leurs utiles suggestions concernant ce

\section{Summary}

Choice and economic weighting of traits in animal selecition.

Two problems of index selection for several traits are discussed, the choice of traits to be included in the aggregate genotype and the economic weighting of these traits. Necessary conditions for including traits and different methods for deriving economic weights are given. The criterion for evaluating the different traits is their genetic economic importance. This value is calculated from the product of the economic weight and the additive genetic standard deviation with the correlated effects added. Examples for swine and cattle are presented and the selection of a combination of several traits to be included in the aggregate genotype is shown. The discussion refers mainly to the distribution of the economic gain due to selection and to the definition of the aggregate genotype. 


\section{Références bibliographiques}

Achilles W., 1968. Die Fleischqualität beim Schwcin als Selektionsmerkmal. Diss. Göttingen.

Adelhelm R., Böckenhoff E., Bischoff T., Fewson D., Rittler A., 1972. Die Leistungsmerkmale beim Rind. Wirtschaftliche Bedeutung und Selektionswürdigkeit. A : Wirtschaftliche Bedeutung der Leistungsmerkmale. Hohenheimer Arbeiten Nr. 64. Ulmer, Stuttgart, 93 pp.

Böckenhoff E., Fewson D., Bischoff T., ig67. Die wirtschaftliche Bedeutung verschiedener Leistungsmerkmale beim Schwein und ihre Auswirkung auf die Zuchtarbeit. I. Mitteilung : Wirtschaftliche Bedeutung der Merkmale. Züchtungskunde, 39, 270-282.

Brehloh B., Schmitten F., 1970. Selektionsindices in ihrer praktischen Anwendung für die Schweinezucht. Züchtungshunde, 42, 93-гот.

Fender M., Böckenhoff E., Zeddies J., Bischoff T., Fewson D., Niebel E., 1976. Neubevechnung von Wirtschaftlichkeitskoeffizienten für die Leistungsmerkmale beim Schwein (En préparation).

Frwson D., 1976. Die wirtschaftliche Bedeutung der Lcistungsmerkmale für die Braunviehzucht. Tierzüchter, 28, 400-403.

Fewson D., Böckenhoff E., Bischoff T., I967. Die wirtschaftliche Bedeutung verschiedener Leistungsmerkmale beim Schwein und ihre Auswirkung auf die Zuchtarbeit. 2. Mitteilung : Auswirkung auf die Zuchtarbeit. Züchtungskunde, 39, 324-33r.

Flock D., I968. Farbhelligkeit im Musculus longissimus dorsi als Selcktionsmerkmal beim Schwein. Fleischwirtschaft, 48, 1362-1365.

Gravert H. O., Rosenhahn E., I965. Welche Kriterien der Mast- und Schlachtleistung von Rindern sind wirtschaftlich wichtig? Züchtungskunde, 37, 244-250.

HARING H. J. F., I972. Zuchtplanung in der Rindersucht aus ökonomischer Sicht. Diss. Göttingen.

HARRIS D. L., I970. Breeding for efficiency in livestock production : Defining the economic objectives. J. Anim. Sci., 30, 860-865.

Hazel L. N., I943. The genetic basis for constructing selection indexes. Gonetics, 28, 476-490.

Hazel L. N., Lush J. L., I942. The efficiency of three methods of selection. J. Hered., 33, 393-399.

Heidhues T., I962. Notwendigkeit und Möglichkeit der Selektion auf mehrere Leistungseigenschaften beim Rind. Züchtungskunde, 34, 99-I I I.

Marguardi O.-W., I974. Selektionskriterien für die niedersächsische Herdbuch-Schweinezucht. Diss. Göttingen.

McClintock A. E., Cunningham E. P., i974. Selection in dual purpose cattle populations : Defining the breeding objective. Anim. Prod., 18, 237-247.

Nieber E., 1974. Methodik zur Zuchtplanung fïr die Reinzucht beim Rind bei Oplimienung nach Zuchtfortschritt und Züchtungsgewinn. Diss. Hohenheim.

Niebel E., Ritrler A., Fewson D., 1972. Die Leistungsmerkmale beim Rind. Wirtschaftliche Bedeutung und Selektionswürdigkeit. B. Selektionswiurdigkeit der Leistungsmerkmale. Hohenheimer Arbeiten Nr. 64, Ulmer, Stuttgart, 93 pp.

Ollivier L., i97i. L'épreuve de la descendance chez le porc Large White français de 19531966. II. Indices de sélection. Ann. Génét. Sél. anim., 3, 367-376.

Pearson A. M., Hayenga M. L., Heifner R. G., Bratzler L. J., Merkel R. A., i97o. Influence of various traits upon live and carcass value for hogs. J. Anim. Sci., 31, 31 8-322.

Rittler A., Werkmeister F., Fewson D., ig66. Untersuchungen über die Prüfung von Bullen auf Mastleistung und Schlachtkörperqualität in zentralen Anstalten. Zü̈htungskunde, 38, I-1o.

Schlote W., 1974. Ökonomische Bewertung von Kriterien der Mastleistung und des Schlachtkörperwertes für die Zuchtplanung beim Rind. Tievzüchtev, 26, 328-330.

Schlote W., Fewson D., I972. Selektionsindizes für die Zuchtwertschätzung beim Rind. Ergebnisse eines Kurses mit Tierzuchtfachlcuten und Studenten. Züchtung gskunde, 44, 35I-359.

Smith H. F., 1936. A discriminant function for plant selection. Ann. Eugenics, 7, 240-250.

Soller Mi., Bar-Anan R., Pasternak H., i 966 . Selection of dairy cattle for growth rate and milk production. Anim. Prod., 8, ro9-II9.

Spahr S. L., I967. Relative economic importance of milk and milk fat percentage in selecting artificially inseminated proven dairy sires. J. Dairy Sci., 50, 847-855.

Zeddies J., I973. Zur ökonomischen Bewertung, Planung und Beurteilung von Rinderzuchtprogrammen. Habil. Göttingen.

Zeddies J., Weniger J. H., I970. Die Leistungssteigerung in der Tierproduktion. I. Mitteilung: Definitionen und ökonomische Auswertungen der Leistungssteigerung. Zü̈chtungskunde, 42, $8 \mathrm{I}-92$. 